\title{
Patent Ductus Arteriosus Device Occlusion in a Patient with Primary Ciliary Dysfunction and Interrupted Inferior Vena Cava: An Interventional Challenge
}

\begin{abstract}
Trans-catheter occlusion of patent ductus arteriosus in patients with interrupted inferior vena cava is possible using antegrade trans-jugular approach or by creating an arterio-venous railroad using femoral venous approach. We report a successful occlusion of a large patent ductus arteriosus in a patient with interrupted inferior vena cava who also had dextrocadia, which offered additional challenges during the occlusion process. Negotiating across the acute bends with a large sized Mullen sheath across the relatively small sized vessels was our major concern, which we were able to accomplish during the process.
\end{abstract}

Keywords: Dextrocardia; Interrupted IVC; Primary ciliary dysfunction; Patent ductus arteriosus; Kartagener Syndrome

Case Report
Volume 10 Issue 2 - 2017
Khurram Akhtar, Mehboob Sultan* and
Adiba Akhtar Khalil
Army Cardiac Center Lahore, AFIC-NIHD Rawalpindi, Pakistan
*Corresponding author: Mehboob Sultan, Army Cardiac
Center Lahore, AFIC-NIHD Rawalpindi, Pakistan, Tel:
00923215129563; Email: drmehboobsultan@gmail.com
Received: November 08, 2017 | Published: November 10,
2017

\section{Introduction}

Trans-catheter occlusion of patent ductus arteriosus (PDA) using antegrade approach through the femoral vein is well established approach commissioned in the catheter lab. However, in patients with interrupted inferior vena cava (IVC), it is not always practicable to close the ductus by employing the usual antegrade approach. While a detailed echocardiographic evaluation of systemic venous drainage is needed prior to any percutaneous intervention procedure, cardiac catheterization is still the gold standard to delineate the systemic venous drainage 1 . Furthermore, it is also required to be aware of any substitute approaches if available, if interrupted IVC comes as a revelation during the course of the catheter procedure.

We report a case of accomplishing PDA closure in a nine-yearold girl diagnosed with "Kartagener Syndrome" (triad of situs inversus, chronic sinusitis and bronchiectasis), interrupted IVC and dextrocardia through the trans-jugular approach.

\section{Case Report}

A nine-year-old girl with bilateral corneal opacities presented to the Paediatric cardiology department for evaluation of her heart murmur. She had prolonged history of ill health with repeated chest infections. She was afebrile at the time of examination. On clinical examination the apex beat was localized in the right fifth intercostal space lateral to the mid-clavicular line. First heart sound was normal in intensity, and the pulmonary component of the second heart sound was loud. There was a continuous murmur heard best in the second right upper second intercostal space.

Chest X-ray revealed cardiomegaly with lung plethora. There were additional patchy infiltrates scattered over the lung fields bilaterally (Figure 1). Transthoracic 2D echocardiography confirmed mirror image dextrocardia with a large conical PDA measuring $07 \mathrm{~mm}$ at its slenderest point in parasternal short axis and suprasternal views and with volume overloaded left atrium \& ventricle apparent on four chamber apical view. A spectral Doppler placed across the ductus in parasternal short axis view, disclosed a continuous flow with peak systolic pressure gradient of $30 \mathrm{mmHg}$.

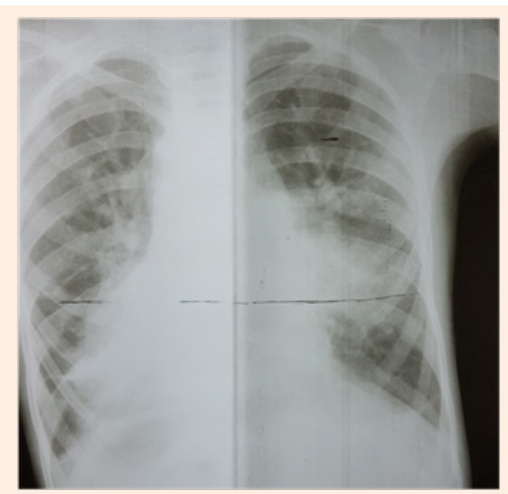

Figure 1: Chest X-ray showed cardiomegaly, and bilateral lung plethora. There were additional patchy infiltrates scattered over the lung fields bilaterally.

Because of the inherent risk of general anaesthesia (GA) in a patient with dysfunctional motility syndrome, we first intended to complete the catheter procedure with mild sedation and a local anaesthetic. However due to the non-cooperation on part of the patient, the procedure had to be accomplished under general anaesthesia. Right femoral vein and artery were cannulated used $5 \mathrm{~F}$ radial sheath. During the procedure, we found the IVC to drain across the azyguous continuation into the right superior vena cava and then track through the dilated coronary sinus in the right atrium Figure 2. 


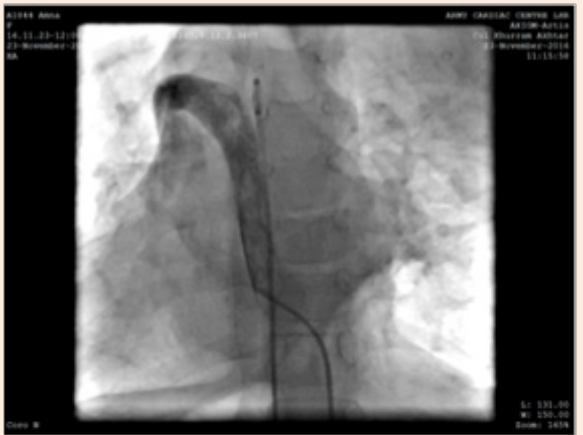

Figure 2: IVC draining across the azyguous continuation into the right superior vena cava and then follow through the dilated coronary sinus in the right atrium.

A decision to carry out the procedure from the left internal jugular vein (accessed with $5 \mathrm{~F}$ radial sheath) was made after confirming the drainage of contrast from the left brachial vein into the right atrium through the SVC Figure 3. During the procedure, a 5F Judkin's right heart coronary catheter was passed over J tip 0.035“ Treumo exchange length guide wire from left internal jugular vein (IJV) to main pulmonary artery (PA) past the right atrium (RA) and right ventricle (RV) and directed into the pulmonary artery. While deliberating the angulations involved in the catheter course and in order to establish an improved support to station the large sized Mullen sheath across the PDA into the descending aorta, we preferred to exchange the $0.035^{\text {" wire with }}$ extra stiff Amplatz wire (AGA Medical Corp, Golden Valley, MN) (Figure 4). Oxygen samples and pressures were then taken.

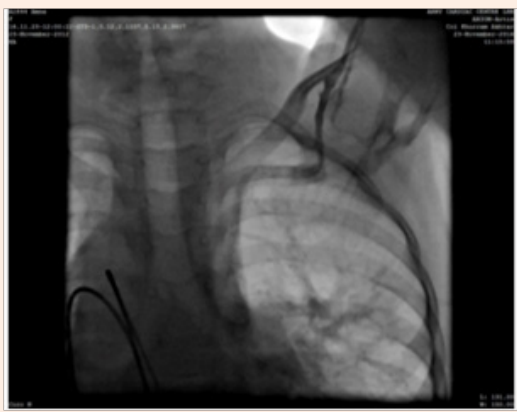

Figure 3: Contrast draining from the left brachial vein into the morphological RA.

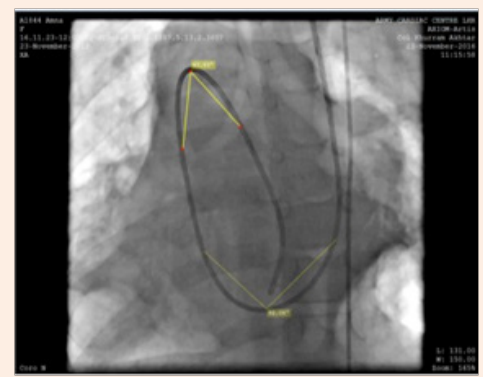

Figure 4: Extra stiff Amplatz 0.035“ wire (AGA Medical Corp, Golden Valley, MN) for improved support and to station the Mullen sheath across the PDA into the descending aorta.
We used a 9F Mullen delivery sheath, which was advanced over and placed in the descending aorta over the super-stiff Amplatz guide wire. In imaging the course of the catheter involved in our case, we cognized that the Mullen sheath had now acquired an almost 90 degree curve from (RA) to pulmonary artery (PA) and another acute curve of 30-40 degree from PA to aorta. However, no struggle was realized while positioning or advancing the device across the sheath (Figure 5).

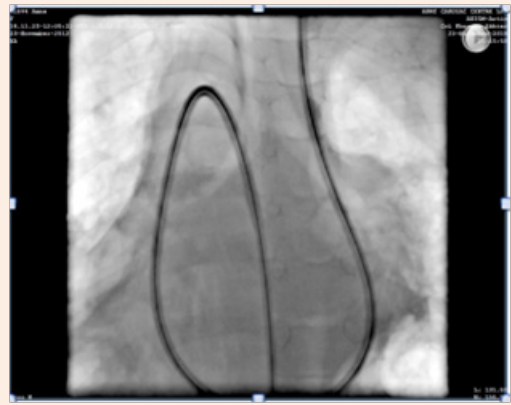

Figure 5: 9F delivery sheath negotiated across the bends.

A duct occluder (Shshma, China) size $14 / 12 \mathrm{~mm} \quad$ w a $\quad \mathrm{s}$ attached to the delivery cable and progressed across the delivery sheath (Figure 6). After confirming the precise location of the device in a lateral angiogram right anterior oblique (RAO) 90 degrees, the device was then disengaged (unscrewed) from the delivery cable. Mild residual flow through the device ("foaming") was however encountered immediately following release. Followup discharge echocardiogram 24 hours after the procedure, disclosed proper placement of the occluder with no residual flow through the device or across the ductus.

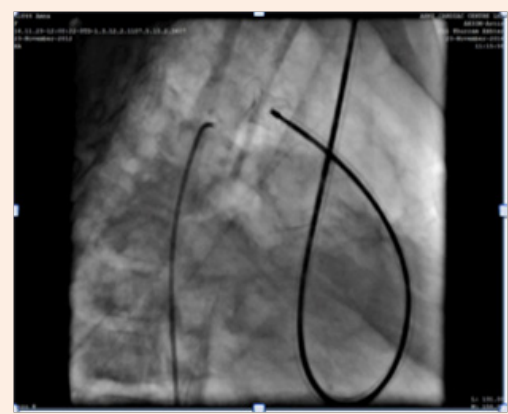

Figure 6: Right lateral angiogram in the descending aorta (RAO 90 degree) confirming proper positioning of the device

\section{Discussion}

Interruption of the IVC is a relatively rare abnormality with an estimated incidence of 1 in 5,000 cases [1]. Our patient not only had interrupted IVC but was also diagnosed as a case of primary ciliary dyskinesia with history of repeated chest infections. Approximately one half of patients with primary ciliary dyskinesia have situs inversus [2]. Normal ciliary beating is necessary for visceral rotation during embryonic development. In patients with primary ciliary dyskinesia, organ rotation occurs as a random event; therefore, half the patients have situs inversus and the other half have normal situs $[2,3]$. 
Trans catheter device closure of ducts is a routine procedure. However trans catheter device closure of a large duct with dextrocardia, interrupted inferior vana cava (IVC) with compromised lungs amid pulmonary hypertension was a considerable challenge in this case. Some of the difficulties expected were the mirror image orientation of the heart, kinking of the catheters across acute bends, possibility of failure to advance a large delivery sheath across the jugular vein in a small child. Options available were to close the ductus through the inferior vena cava by establishing an arterio-venous railroad $[1,4]$ or using the left internal jugular vein for accessing the venous side [5]. We chose the later approach and were able to successfully negotiate through the acute bends without encountering significant problem and were thus able to occlude the ductus through the transjugalar approach.

\section{References}

1. Akhtar S, Samad SM, Atiq M (2010) Transcatheter closure of a patent ductus arteriosus in a patient with an anomalous inferior vena cava. Pediatr Cardiol 31(7): 1093-1095.

2. Tuberk BK, Zur KM (1933) Pathogenese der bronchiectasien. Mitteilung:bronchiectasien bei situs viscerum inversus 83: 498-501.

3. Patel NH, Madan TH, Panchal AM, Thakkar BM (2009) Percutaneous closure of patent ductus arteriosus through internal jugular vein in patient with interrupted inferior vena cava. Ann Pediatr Cardiol 2(2): 162-164

4. Tefera E, Cañete R B (2014) Percutaneous closure of patent ductus arteriosus in interrupted inferior caval vein through femoral vein approach. Ann Pediatr Cardiol 7(1): 55-57.

5. Aggarwal N, Agarwal M, Joshi R, Joshi RK (2015) Device closure of patent ductus arteriosus in interrupted inferior vena cava. Indian Heart J 67 Suppl 3: S85-S87. 